\title{
Nanoparticle growth by collection of ions: orbital motion limited theory and collision- enhanced collection
}

Iris Pilch, L. Caillault, T. Minea, Ulf Helmersson, Alexey Tal, Igor Abrikosov, Peter Münger and Nils Brenning

Journal Article

\section{Tweet}

N.B.: When citing this work, cite the original article.

Original Publication:

Iris Pilch, L. Caillault, T. Minea, Ulf Helmersson, Alexey Tal, Igor Abrikosov, Peter Münger and Nils Brenning, Nanoparticle growth by collection of ions: orbital motion limited theory and collision-enhanced collection, Journal of Physics D, 2016. 49(39), pp.395208.

http://dx.doi.org/10.1088/0022-3727/49/39/395208

Copyright: IOP Publishing: Hybrid Open Access

http://www.iop.org/

Postprint available at: Linköping University Electronic Press

http://urn.kb.se/resolve?urn=urn:nbn:se:liu:diva-132204

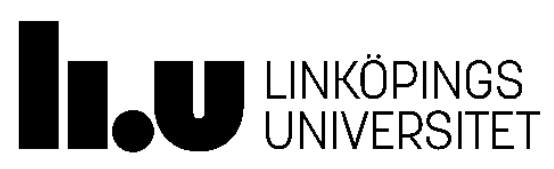




\section{Nanoparticle growth by collection of ions: orbital motion limited theory and collision-enhanced collection}

I. Pilch, ${ }^{1}$ L. Caillault, ${ }^{2}$ T. Minea, ${ }^{2}$ U. Helmersson, ${ }^{3}$ A. A. Tal, ${ }^{4,5}$ I. A. Abrikosov,, ${ }^{4,5}$ E. P. Münger, ${ }^{4}$ and N. Brenning3,6

1) Thin Film Physics Division, Department of Physics, Chemistry, and Biology (IFM), Linköping University, SE-581 83 Linköping, Sweden

2) Laboratoire the Physique de Gaz et de Plasmas, UMR 7198 CNRS, Université Paris-Sud 91405 Orsay Cedex, France

3)Plasma \& Coatings Physics Division, IFM-Materials Physics, Linköping University, SE-581 83 Linköping, Sweden

4) Theory and Modeling Division, IFM-Material Physics, Linköping University, SE-581 83 Linköping, Sweden

5) Materials Modeling and Development Laboratory, National University of Science and Technology “MISIS," 119049 Moscow, Russia.

6) KTH Royal Institute of Technology, School of Electrical Engineering, Division of Space and Plasma Physics, SE-10 044 Stockholm, Sweden

PACS numbers: _

Electronic address: iripi@ifm.liu.se

\section{Abstract}

The growth of nanoparticles in a plasma is modeled for situations where the growth is mainly due to collection of ions of the growth material. The model is based on classical orbit motion limited (OML) theory with the addition of collision-enhanced collection (CEC) of ions. The limits for this type of model are assessed with respect to three not include processes: evaporation of the growth material, electron field emission, and thermionic emission of electrons. It is found that both evaporation and thermionic emission can be disregarded below a temperature that depends on the nanoparticle material and on the plasma parameters, for copper in our high-density plasma this limit 
is about $1200 \mathrm{~K}$. Electron field emission can be disregarded above a critical nanoparticle radius, in our case around $1.4 \mathrm{~nm}$. The model is benchmarked, with good agreement, to the growth of copper nanoparticles from a radius of $5 \mathrm{~nm}$ to $20 \mathrm{~nm}$ in a pulsed power hollow cathode discharge. Ion collection by collisions contributes with approximately $10 \%$ of the total current to particle growth, in spite of the fact that the collision mean free path is four orders of magnitude longer than the nanoparticle radius.

\section{Introduction}

The charge of a nanoparticle is defined by the floating potential that a nanoparticle attains in a plasma, where the floating potential is determined by an equilibrium of electron and ion currents to and from the nanoparticle. This leads in low temperature plasmas typically to negatively charged nanoparticles as the electron temperature is much larger than the ion temperature. For the growth of nanoparticles the floating potential is an important quantity because in case that all nanoparticles are negatively charged, coagulation of nanoparticles cannot occur any longer, and also because they will selectively collect positive ions rather than neutrals and negative ions. This offers a route for fast growth of nanoparticles if large amounts of positive ions can be created [1].

In order to estimate the electron and ion currents to a nanoparticle, standard probe theory can be used like the orbital motion limited (OML) theory [2, 3], when the boundary conditions are fulfilled. The OML theory was originally proposed to provide correct estimates when the shielding length is much larger than the nanoparticle radius and collisions are rare. A more detailed description requires that the mean free path length $l_{\text {coll }}$ of ions is much larger than an effective OML collision radius: $l_{\text {coll }} \gg r_{O M L} \approx$ $r_{N P}\left(\left|e \Phi_{N P}\right| / k_{B} T_{i}\right)^{1 / 2}$ [2], with the nanoparticle radius $r_{N P}$, the electron charge $e$, the nanoparticle potential $\Phi_{N P}$, the Boltzmann constant $k_{B}$, and the ion temperature $T_{i}$. However, it was shown that even few collisions can lead to an increase of the ion current $[4,5,6,7,8,9]$. We here operate with a typical gas pressure of the order of $100 \mathrm{~Pa}$, where the mean free path length for ions at the gas temperature is about $100-200 \mu \mathrm{m}$. For nanoparticles below $100 \mathrm{~nm}$ diameter the condition above is then satisfied by two to three orders of magnitude. Nevertheless, we will show that even these very few collisions lead 
to an increase of the ion current, and thereby to an increase of the growth rate of ioncollecting nanoparticles.

A couple of theories have been presented that are based on the OML theory but include collision-enhanced collection (CEC) of ions, e.g. $[8,6,10]$. There, the ion current is the sum of the OML contribution and the current due to collision within a sphere of a capture radius. Due to the large volume of the capture sphere, CEC can contribute and lead to an increased ion current even when the mean free path length is much larger than the capture radius. A model to cover a wide range of pressures was presented by Gatti and Kortshagen $[4,11]$ where the ion current has three contributions: the standard OML contribution, CEC ion collection, and a hydrodynamic ion current that becomes important at high pressure.

In this contribution, a model is presented for the growth of nanoparticles by collection of ions in a given plasma environment. Specific attention are given to access the range of applicability of the model and to an increased ion current caused by collisions. For small nanoparticle radii and at high temperatures, evaporation, electron field emission (EFE) and thermionic emission (TIE) put limits to the applicability of this type of model, and are therefore assessed in this work. The maximum temperature at which evaporation can be neglected and a minimum nanoparticle size until which electron field emission is negligible are estimated. An enhanced growth of nanoparticles through an increase of the ion current due to collisions is demonstrated and compared to previous experimental results [1]. For a numerical example we use a reference case with typical parameters of pressure $p_{A r}=107 \mathrm{~Pa}$, ion and gas temperature $T_{i}=T_{A r}=300 \mathrm{~K}(26 \mathrm{meV})$, argon ion density $n_{\mathrm{Ar}^{+}}=3 \cdot 10^{18} \mathrm{~m}^{-3}$, copper ion density $n_{\mathrm{Cu}^{+}}=3 \cdot 10^{18} \mathrm{~m}^{-3}$, electron density $n_{e}=$ $6 \cdot 10^{18} \mathrm{~m}^{-3}$, electron temperature $T_{e}=1 \mathrm{eV}$ and nanoparticle sizes in the range of $r_{N P}=$ $5 \mathrm{~nm}$ to $20 \mathrm{~nm}$.

\section{Theoretical Description}

For studying the growth of nanoparticles by collection of ions, the currents to a nanoparticle as function of plasma parameters are calculated. The model is based on particle charging through orbit motion limited (OML) theory [2, 3] and extended by collision-enhanced collection (CEC) $[4,11]$. The extended model follows the approach of Gatti and Kortshagen [4] but is here limited to a low collisionality regime (to be defined 
below), in which a hydrodynamic contribution to the ion current can be neglected. It will hereafter be termed OML/CEC model. The processes addressed in the presented work is a subset of a more complete nanoparticle growth model that is under development, and which is shown in Fig. 1. Solid red lines mark parameters and processes that are included in the OML/CEC model with a full treatment, and dashed yellow lines mark parameters and processes that are treated only as needed to assess the limits of the applicability of the OML/CEC model. Furthermore, we only determine the steady-state potential for a given set of parameters, i. e., statistical charging effects are not considered. At smaller nanoparticle sizes, below about $r_{N P}=5 \mathrm{~nm}$, discrete charging events and charge fluctuations become significant and a statistical charging approach is needed [12]. Due to charge fluctuations a small portion of nanoparticles may even become positively charged [12]. A model for growth of nanoparticles in an electronegative silane plasma was presented in Ref. [13]. For our reference case, the floating potential is around $\emptyset_{N P} \approx$ $-3 V$, which corresponds to a charge of about $Q=-10 e$ for a nanoparticle with radius $r_{N P}=5 \mathrm{~nm}$. Using the statistical charging approach described in Ref. [12], the standard deviation of the charge is around $\pm 2 e$. Hence, it is a reasonable to not consider statistical charging effects for estimating the limits of applicability of the model for nanoparticles larger than $5 \mathrm{~nm}$, and a detailed analysis of statistical charging for nanoparticles smaller than $5 \mathrm{~nm}$ is beyond the scope of this contribution.

\subsection{Orbital Motion Limited Theory and Collision-Enhanced Collection}

The types of collisions with nanoparticles that are included in the OML/CEC model are sketched in Fig. 2(a) together with their effective cross sections. Electrons are repelled by the negative potential and have the smallest effective cross section. Neutral atoms are regarded as collected when their trajectories intersect with the geometrical cross section of a nanoparticle: $\sigma_{0}=\pi r_{N P}^{2}$. Positive ions have much larger cross sections representing OML collection and CEC.

The electron-charging current to a negatively charged nanoparticle is described with standard OML theory [2] and given as

$$
I_{e}=-\frac{1}{4} e n_{e} v_{e, t h} \sigma_{0} \exp \left(-\frac{\left|e \emptyset_{N P}\right|}{k_{B} T_{e}}\right),
$$


with the electron charge $e$, the electron density $n_{e}$, the electron temperature $T_{e}$, the thermal velocity $v_{e, t h}=\sqrt{8 k_{B} T_{e} / \pi m_{e}}$, the nanoparticle potential $\emptyset_{N P}$, and the Boltzman constant $k_{B}$.

We define the low collisionality regime as where hydrodynamic effects on the ion current (e.g., see Ref. [4]) are negligible. Under this assumption, the ion-charging current to a nanoparticle is given by

$$
I_{i}=I_{O M L}+I_{C E C}
$$

In the absence of collisions, the OML process gives the ion collection current

$$
I_{O M L, \text { coll.free }}=\sum \frac{1}{4} q_{i} \sigma_{0} n_{i} v_{i, t h}\left(1+\frac{\left|e \Phi_{N P}\right|}{k_{B} T_{i}}\right),
$$

where $q_{i}$ is the charge of the ion, $T_{i}$ the ion temperature, $n_{i}$ the ion density, $v_{i, t h}=$ $\sqrt{8 k_{B} T_{i} /\left(\pi m_{i}\right)}$ the thermal ion velocity, and $\phi_{N P}$ the (negative) nanoparticle potential, i.e., floating potential. The summation in Eq. (3) is over the different types of ions, for our reference case copper $\left(\mathrm{Cu}^{+}\right)$and argon $\left(\mathrm{Ar}^{+}\right)$ions. An effective OML collision radius can be defined from Eq. (3) as $r_{O M L}=r_{N P}\left(1+\left|e \Phi_{N P}\right| / k_{B} T_{i}\right)^{1 / 2}$.

In the presence of collisions, $I_{O M L}$ becomes lower since some of the ions that are directed towards the OML capture cross section will, as shown in Fig. 2(a), collide inside the capture radius. These ions must be subtracted from the OML current, giving

$$
I_{O M L}=\sum \exp \left(-2 \alpha \frac{r_{c a p}}{l_{\text {coll }}}\right) 4 q_{i} \sigma_{0} n_{i} v_{i, t h}\left(1+\frac{\left|e \Phi_{N P}\right|}{k_{B} T_{i}}\right)
$$

The factor $\alpha=1.22$ accounts for the collision probability of ions that pass inside the capture radius $r_{\text {cap }}$ [4], which is defined as the locus where the potential energy of an ion-according to the Debye-Hückel potential-equals its average kinetic energy $E_{k i n}$ after a collision. Ions that collide inside this capture radius will become trapped in closed 
orbits and eventually intercept the nanoparticle, although this might require several collisions. The capture radius is given as [4]

$$
r_{c a p}=\frac{r_{N P}\left|e \emptyset_{N P}\right|\left(1+\frac{r_{N P}}{\lambda_{D}}\right)}{E_{k i n}+\left|e \emptyset_{N P}\right| \frac{r_{N P}}{\lambda_{D}}} .
$$

The two terms in Eq. (5) that include the shielding length $\lambda_{\mathrm{D}}$ account for screening: further away than approximately $\lambda_{\mathrm{D}}$, ions are screened from the nanoparticle potential and cannot be trapped in orbits around it. These two terms become small, and screening therefore negligible, at combinations of low plasma density and small nanoparticles. The condition is that the terms in the denominator of Eq. (5) obey $E_{k i n} \gg\left|e \emptyset_{N P}\right| \frac{r_{N P}}{\lambda_{D}}$. In this range $\lambda_{D} \gg r_{c a p}$ and the capture radius becomes independent of $\lambda_{D}$ :

$$
r_{c a p}=\frac{r_{N P}\left|e \emptyset_{N P}\right|}{E_{k i n}}
$$

Typical length scales are shown in Fig. 2(b) and indicate that we cannot make this simplification for our reference case. A value for the shielding length $\lambda_{D}$ is therefore needed. An appropriate choice for the Debye length is discussed in literature $[14,15]$, and some studies address the question $[16,17]$. In Eq. (5), the Debye length $\lambda_{D}$ was denoted without specification if it is the electron Debye length $\lambda_{D e}$, the ion Debye length $\lambda_{D i}$, or the linearized Debye length $\lambda_{D l}=\left(\lambda_{D e}^{-2}+\lambda_{D i}^{-2}\right)^{-1 / 2} \approx \lambda_{D i}$. For the reference case of this work, the two extreme values $\lambda_{D e}$ and $\lambda_{D i}$ are shown in Fig. 2(b). In the following calculations, we will use a cutoff radius $r_{c}$ for specifying the Debye length as proposed by Hutchinson and Haakonen [16] for cases like ours: an ion-attracting spherical particle 
that is stationary with respect to a surrounding Maxwellian plasma, where $\lambda_{D} \gg r_{N P}$, and when trapped ions dominate the shielding:

$$
\lambda_{D}=r_{C} \approx\left(\frac{r_{N P} T_{i}}{\lambda_{D e_{e}}}\right)^{\frac{1}{5}} \lambda_{D e}
$$

The CEC ion current is taken from Gatti and Kortshagen [4], with the difference that we have rewritten their expressions to include the collision probability in the expression for $I_{C E C}$ :

$$
I_{C E C}=\sum 2 \alpha \frac{r_{c a p}}{l_{\text {coll }}} \exp \left(-2 \alpha \frac{r_{c a p}}{l_{\text {coll }}}\right) \pi q_{i}\left(\alpha r_{c a p}\right)^{2} n_{i} v_{i, t h}
$$

As can be seen in Fig. 2 (b), our collision mean free path is about two orders of magnitude larger than the capture radius and one would intuitively expect the effect of collisions to be negligible. However, a typical value in the present experiment is $r_{c a p} \approx 100 r_{N P}$ and a capture volume (i. e., the volume inside $r_{c a p}$ ) that is around $10^{6}$ times the volume of the nanoparticle itself. The large number of ions inside the capture volume is the reason why $I_{C E C}$ can become significant even when only a small fraction of them collide and are captured.

In the evaluation of Eq. (5), we assume that the ions after a collision have an average kinetic energy $E_{k i n}=\left(3 k_{B} T_{n}\right) / 2$ of the neutral gas, with $T_{n}=300 \mathrm{~K}$. The collision mean free path is given by $l_{\text {coll }}=1 /\left(n_{n} \sigma_{\text {coll }}\right)=k_{B} T_{n} /\left(p \sigma_{\text {coll }}\right)$ with the neutral gas density $n_{n}$, the neutral gas temperature $T_{n}$, the gas pressure $p$, and the elastic (momentum exchange) collision cross section $\sigma_{\text {coll }}$ for each ion specie with the background gas. The collision cross section for argon ions in argon is well studied and has a large contribution from resonant charge exchange collisions, and we use an average value for the ion-neutral collisions between argon atoms and ions of $\sigma_{A r^{+}}=40.9 \cdot 10^{-20} \mathrm{~m}^{2}$ given by Galli [11]. For copper ions-which is the second ion specie and which causes nanoparticle growth - charge exchange is energetically impossible and the collision cross section with argon atoms is less well known, particularly at the low ion energies of interest here. For this cross section we make the following estimate based on scaling from the argon cross section above. We first note that the ion mobility is related to the elastic cross section through the relations $\mu_{i}=e D_{i} / k_{B} T_{i}, D_{i}=3 \pi /(16 \sqrt{2}) \cdot v_{i, t h} l_{\text {coll }}$, and $l_{\text {coll }}=1 /\left(n_{g} \sigma_{\text {coll }}\right)$. From these relations follows the proportionality $\sigma_{\text {coll }} \propto \frac{1}{\mu_{i}}$. For 
the scaling we chose mobilities for the case of zero drift speed $(E / p \rightarrow 0)$ and a gas temperature of $300 \mathrm{~K}$. Chanin and Biondi [18] have summarized measured thermal (300 K) mobilities in Ar of the metal ions $\mathrm{Li}^{+}, \mathrm{Na}^{+}, \mathrm{Rb}^{+}, \mathrm{Cs}^{+}$, and $\mathrm{Hg}^{+}$. They all fall along a simple curve, when plotted against the mass number. Assuming that the mobility for $\mathrm{Cu}^{+}$falls on the same curve, and scales with the mobility for $\mathrm{Ar}^{+}$in the same way (for the case 300 $\mathrm{K}, E / p \rightarrow 0$ ), we obtain $\sigma_{\mathrm{Cu}^{+}}=28 \cdot 10^{-20} \mathrm{~m}^{2}$.

A time-average floating potential $\Phi_{N P}$ is obtained from Eqs. (1) to (8) using the steadystate condition

$$
I_{O M L}+I_{C E C}+I_{e}=0
$$

The total growth rate of a nanoparticle is given by the sum of the ion and neutral fluxes of the growth material:

$$
\frac{d r_{N P}}{d t}=\left(\frac{I_{M^{+}, O M L}^{+I_{M^{+}, C E C}}}{q_{i}}+n_{M} v_{M, t h} \sigma_{0}\right) \cdot\left(\frac{m_{M}}{4 \pi r_{N P}^{2} \rho_{M}}\right)
$$

where $v_{M, t h}$ is the thermal velocity of the metal atoms (i.e., copper), $m_{M}$ the mass of the metal atom (ion) and $\rho_{M}$ the bulk density of the metal. The model follows the growth of nanoparticles, and the variation of their potential with time, in an environment that has a known development in time of the gas and plasma parameters. Nanoparticles with an initial radius are used as seeds, and the external parameters that are shown to the left in Fig. 1 are set: $n_{A r}, n_{A r^{+}}, n_{M}, n_{M^{+}}, n_{e}=n_{A r^{+}}+n_{M^{+}}, T_{e}, T_{i}$, and $T_{g}$. The outputs of the model are the radius $r_{N P}$ and the potential $\emptyset_{N P}$ of a nanoparticle as function of time.

\section{Limits of applicability}

We will here discuss the conditions of applicability of the OML/CEC model. At high gas pressures hydrodynamic effects influence the CEC ion current to the nanoparticles. For nanoparticles that are very small and for nanoparticle with a high temperature, electron field emission (EFE), thermionic emission of electrons (TIE), and desorption can become important. These effects limit the applicability of the present OML/CEC model to parameters which are here assessed in terms of gas pressure, nanoparticle temperature and nanoparticle radius. The estimations are made assuming single nanoparticles. In case of a high nanoparticle density $\left(n_{N P}\right)$, charge reduction can take place. A parameter for describing this effect is the so-called Havnes parameter $\left(P=4 \pi r_{N P} \lambda_{D e}^{2} n_{N P}\right)$, and charge 
reduction can be neglected for $P \ll 1[19,20,21]$. For our reference case $\left(T_{e}=1 \mathrm{eV}, n_{e}=\right.$ $6 \cdot 10^{18} \mathrm{~m}^{-3}$ ) and a nanoparticle radius of $10 \mathrm{~nm}$, this is valid for nanoparticle densities of $n_{N P}=10^{14} \mathrm{~m}^{-3}\left(P=10^{-4}\right)$. For larger nanoparticle densities of $n_{N P}=10^{17} \mathrm{~m}^{-3}(P=0.1)$, charge reduction due to high nanoparticle densities should be taken into consideration.

\subsection{Limit of applicability in gas density: hydrodynamic effects}

The OML/CEC model was derived in section 2.1 assuming any hydrodynamic effects (see e.g. [4]) on the ion current to the nanoparticle to be negligible. The question is if the

current $I_{C E C}$ of Eq. (8) correctly accounts for the collection of all ions that make a "first collision" inside the capture sphere. In the CEC description, these ions become trapped in closed orbits, and a sequence of collisions then scatter them into successively lower orbits until they hit the nanoparticle. This description breaks down at so high gas density that multiple collisions significantly influence the closed orbits. At very high pressure, in the hydrodynamic range, collisions actually slow down the ion collection process which must be described in terms of diffusion and mobility [4].

The purely hydrodynamic description holds under the condition $l_{\text {coll }} \ll r_{\text {cap }}$, i.e., when the gas pressure is so high that the mean free path $l_{\text {coll }}$ is much smaller than the capture radius $r_{c a p}$ of Eq. (5). As a condition for hydrodynamic effects to be negligible, we propose the opposite condition: $l_{\text {coll }} \gg r_{\text {cap }}$. The typical length scales in our experiment in Fig. 2(b) shows that we are in a range where this condition holds.

\subsection{Limit of applicability in temperature, I: desorption}

It is known that the temperature of a nanoparticle in a plasma can be higher than the ambient gas temperature [22]. A higher temperature of a nanoparticle can lead to an increased evaporation rate and therewith limit the growth of a nanoparticle. To estimate when evaporation becomes important it is necessary to estimate the temperature at which the thermal desorption rate of atoms becomes significant in comparison to collection of ions [Eq. (10)].

Molecular dynamics calculations of the copper evaporation rate from particles of different sizes and temperature have been calculated and are shown in Fig. 3. The calculations were performed in LAMMPS [23] with embedded-atom-method potentials [24]. The statistics of the evaporation events was accumulated during $400 \mathrm{~ns}$ of the simulation time for each 
simulation. We performed five simulations for each temperature value in order to estimate the error. It should be mentioned that for the lower temperatures the statistics are less good and errors larger as compared to higher temperatures. To improve on statistics for the low temperature calculations or to go to even lower temperatures, are unfortunately very computationally expensive due to low evaporation rates requiring very long simulations. Moreover, the atomic interaction potential is fitted to bulk properties and might be less accurate for the smallest ( 100 atom) clusters. Both reasons could be responsible for the deviation from the linear behavior for small clusters.

The calculated evaporation rates are compared to evaporation rates for bulk material of copper using [25]

$$
\Gamma_{e v, b u l k}=\frac{\beta}{\sqrt{2 \pi m_{C u} k_{B}}} \cdot \frac{p_{v}}{\sqrt{T}} \approx 4.38 \cdot 10^{25} \cdot \frac{p_{v}}{\sqrt{T}},
$$

where $\beta$ is a constant between 0 and $1, p_{v}$ the vapor pressure in Torr and $T$ the temperature of the material in $\mathrm{K}$. The evaporation rates are in units of $\mathrm{m}^{-2} \mathrm{~s}^{-1}$ with a maximum rate of evaporation when $\beta=1$. For calculating the bulk evaporation rates, the data for the vapor pressure and temperatures were taken from Ref. [26].

The general trend is shown in Fig. 3 with evaporation rate for the smallest nanoparticle (108 atoms) being about one order of magnitude higher as compared to the evaporation rate from bulk copper. For the reference case, the deposition rate of ions is of the order of $10^{25} \mathrm{~m}^{-2} \mathrm{~s}^{-1}$, which is lower than the data accessible with molecular dynamics calculations. However, a comparison with the bulk evaporation rates using data presented in Ref. [26] shows that evaporation is compensated by deposition for temperatures below $1200 \mathrm{~K}$ $\left[\Gamma_{e v, \text { bulk }}\left(T=1205 \mathrm{~K}, p_{v}=10^{-5}\right.\right.$ Torr $\left.)=1.27 \cdot 10^{19} \mathrm{~m}^{-2} \mathrm{~s}^{-1}\right]$.

\subsection{Limit of applicability in temperature, II: thermionic electron emission}

First, calculate the thermionic emission from bulk material at zero electric field, when field emission can be neglected. The current density $\left(\mathrm{A} / \mathrm{m}^{2}\right)$ of thermionic emission can then be estimated using the Richardson-Dushman equation [27]:

$$
J_{T I E}=\frac{4 \pi m_{e} e}{h^{3}}\left(k_{B} T_{N P}\right)^{2} \exp \left(-\frac{\emptyset_{W F}}{k_{B} T_{N P}}\right)
$$


with the electron mass $m_{e}$, the Planck constant $h$, the work function $\emptyset_{W F}$, and the nanoparticle temperature $T_{N P}$. The current is obtained from the current density by multiplication with the area: $I_{T I E}=\pi r_{N P}^{2} J_{T I E}$.

The thermionic electron emission current density (black solid line) is shown in Fig. 4. Two limiting cases were calculated using OML theory: (a) a low density direct current (dc) discharge $\left(n_{e}=10^{15} \mathrm{~m}^{-3}, T_{e}=1 \mathrm{eV}\right)$ and (b) a high power pulsed plasma $\left(n_{e}=10^{20} \mathrm{~m}^{-3}\right.$, $T_{e}=3 \mathrm{eV}$ ). The current density was calculated for a nanoparticle radius of $10 \mathrm{~nm}$. In case of the pulsed discharge, thermionic emission is small compared to the OML currents for temperatures below approximately $2400 \mathrm{~K}$. For the dc case, the nanoparticle temperature needs to be less than $1600 \mathrm{~K}$.

In summary, the requirement of negligible evaporation limits the range of applicability of the OML/CEC model to the range $T_{N P}<1200 \mathrm{~K}$. In this temperature range we expect desorption and thermionic electron emission to be negligible.

\subsection{Limit of applicability in size: electron field emission}

The total electron emission caused by both electron field emission (EFE) and thermionic emission (TIE) has been estimated for copper nanoparticles using the theory of Murphy and Good for bulk material [28]. They proposed an analytical expression for the electron current density which depends on the temperature of the material, here $T_{N P}$, the local electric field at the surface, and the intrinsic properties of the emissive material such as work function $\Phi_{W F}$ and Fermi energy $E_{F}$. The Fermi energy for copper was set to $E_{F}=7.0$ $\mathrm{eV}$ [29]. The work function depends on the crystallographic orientation and varies between 4.5 to $5.0 \mathrm{eV}$ [29]. In this work a mean value of $\Phi_{W F}=4.7 \mathrm{eV}$ has been used. For small nanoparticle sizes, below $5 \mathrm{~nm}$, there is a reduction in work function [30] which has been assessed in a separate publication by density functional theory (DFT) calculations and shown to be negligible for our sizes $r_{N P} \geq 5 \mathrm{~nm}$ [31]. It is assumed that the nanoparticle is in thermal equilibrium so that the temperature inside the nanoparticle is constant and uniform. This should be fulfilled at steady state conditions since copper is a good thermal conductor and the conduction length scale is smaller than the particle size. The temperature range was chosen to be between 300 and $1358 \mathrm{~K}$. The local electric field is assessed by considering perfect spherical, charged particles with radii between $1.0 \mathrm{~nm}$ 
to $41.5 \mathrm{~nm}$. The electric field $E_{N P}$ at the surface of a nanoparticle is given by $E_{N P}=$ $\phi_{N P} / r_{N P}$. For thermionic emission and electric field emission processes, however, the contribution to this field from one electron has to be subtracted since the emitted electron cannot exert a force upon itself. The relevant field strength for electron emission becomes

$$
E_{N P, e m}=\frac{\left|\phi_{N P}\right|-\frac{e}{4 \pi \varepsilon_{0}}}{r_{N P}}=\frac{\left|\phi_{N P}\right|}{r_{N P}}-\frac{e}{4 \pi \varepsilon_{0} r_{N P}} .
$$

This correction is important for nanoparticles where the charge number is low, as highlighted by the special case of charge number 1 where $E_{N P, e m}=0$, and where there is no field emission. The curvature effect of the nanoparticle surface has been neglected in the description of the potential; also this becomes more important for smaller nanoparticles. The combined error from the approximations above (which are implicitly made in applying the bulk matter theory of Murphy and Good to nanoparticles) is assessed to be negligible above $r_{N P}=20 \mathrm{~nm}$, and to give an overestimate of $I_{E F E}$ by at most a factor $10^{2}$ for smaller sizes. Plasma screening of the electric field is neglected in the emission process since it occurs through a potential barrier with a thickness of the order of $r_{N P}$ which is one to two orders of magnitude smaller than the shielding length $\lambda_{D}$, see Fig. 2(b).

For the calculation of the total electron emission current $I_{e m}=I_{E F E}+I_{T I E}$, we limit our estimation to a temperature range below which thermionic emission $I_{T I E}$ can be neglected in agreement with the Richardson-Dushman equation. Further, we will restrict our estimations to calculations for a temperature of $300 \mathrm{~K}$ and denote them as $I_{E F E}$. Since the electron emission current is due to field emission, it is a function of the emission-relevant electric field strength of Eq. (13). For multiple charged nanoparticles, the second term in the nominator is small in the sense $\frac{e}{4 \pi \varepsilon_{0}} \ll\left|\phi_{N P}\right|$, and the electric field can be approximated as $E_{N P, e m} \cong E_{N P}=\left|\phi_{N P}\right| / r_{N P}$. This makes it suitable to plot the electron emission current as a function of the normalized radius $\frac{r_{\mathrm{NP}}}{\left|\emptyset_{\mathrm{NP}}\right|}$, which is shown in Fig. 5. The data points represent calculations for different nanoparticle potentials in the range of -4 to $-15 \mathrm{~V}$ and with different radii. The resulting currents are plotted as functions of $r_{\mathrm{NP}} /\left|\emptyset_{\mathrm{NP}}\right|$. In the logarithmic scale, a close to linear relationship between the electron 
emission current and the normalized potential is found for each potential, but changes in the nanoparticle potential lead to an approximate parallel shift of the entire curve.

The solid curve in Fig. 5 shows an analytical approximation obtained by making a numerical fit to the case $\emptyset_{N P}=-7 \mathrm{~V}$, with $r_{N P}$ in nm,

$$
I_{\mathrm{EFE}}=4.9\left(\frac{r_{N P}}{\left|\emptyset_{N P}\right|}\right)^{2} \exp \left(-74.2 \cdot \frac{r_{N P}}{\left|\emptyset_{N P}\right|}\right) .
$$

Inspection of Fig. 5 shows that this fit, for other values of the potential than $-7 \mathrm{~V}$, gives errors by up to an order of magnitude. The reason for this is that $I_{\mathrm{EFE}}$ is an extremely steep function of the normalized radius. It is therefore too simplified to accurately predict nanoparticle potentials in the range where electron field emission is important. Nevertheless, it can still be used for quite good estimates of for which nanoparticle sizes EFE can be safely neglected. The dashed lines in Fig. 5 are drawn in a range where $I_{\mathrm{EFE}} \sim I_{O M L}+I_{C E C}$ for our reference case. They show that a change with only about $15 \%$ in the normalized radius gives a change of two orders of magnitude in the electron emission current. A requirement of the type $I_{\mathrm{EFE}} \ll I_{O M L}+I_{C E C}$, with a given ion current $I_{O M L}+$ $I_{C E C}$ in a known plasma, therefore gives a constraint on the normalized radius that is robust also against quite large uncertainties in $I_{\mathrm{EFE}}$.

The question is the following: in a given plasma environment, as defined by $n_{e}$ and $T_{e}$, what is the critical radius $r_{N P, c r i t}$ above which electron field emission can be neglected, i.e. $I_{\mathrm{EFE}} \ll I_{O M L}+I_{C E C} ?$

We assume to be in the OML regime (which has to be motivated a posteriori). Here, we use the relation between $\emptyset_{N P}$ and $T_{e}$ as [1]

$$
\emptyset_{N P}=-K_{1} T_{e}
$$

Where $T_{e}$ is in $\mathrm{eV}$ and $K_{1}$ is a constant that depends on the ion mass and the $T_{e} / T_{i}$ ratio. The OML ion current is [1]

$$
I_{O M L}=\frac{1}{4} e n_{e} v_{i, t h} \pi r_{N P}^{2}\left(1+K_{1} \frac{T_{e}}{T_{i}}\right)
$$

where $r_{N P}$ is in units of $\mathrm{m}$. For a typical discharge plasma with $T_{i}=T_{n}=0.026 \mathrm{eV}(300$ $\mathrm{K})$, and $T_{e} \gg T_{n}$, this gives 


$$
I_{O M L} \cong 4.84 \times 10^{-36} v_{i, t h} K_{1} n_{e} r_{N P}^{2} T_{e}
$$

Let us introduce a constant $K_{2}$ to quantify our safety margin for negligible field emission, i.e., how much smaller than the current $I_{O M L}$ we require the field emission current $I_{E F E}$ to be:

$$
K_{2}=\frac{I_{E F E}}{I_{O M L}}
$$

Combining Eqs. (14) to (18) gives the critical radius (here converted to units of $\mathrm{nm}$ ) as function of $T_{e}$ and $n_{e}$,

$$
r_{N P, \text { crit }}\left(K_{2}\right)=-0.014 K_{1} T_{e} \ln \left(9.88 \times 10^{-37} K_{1}^{3} K_{2} v_{i, t h} n_{e} T_{e}^{3}\right),
$$

which we write as a function of $K_{2}$ in order to emphasize that it is a function of an arbitrarily chosen constant.

Now let us rewrite this result to suit our special case. As a condition for how negligible EFE current shall be, we chose $K_{2}=10^{-4}$. We regard this factor to be enough to cover both theoretical uncertainties at small $r_{N P}$ in the Murphy and Good theory (the errors in the locations of the circles in Fig. 4), and the deviations from these circles of the approximation of Eq. (14) (the solid line in Fig. 4). We also use $v_{i, t h}$ for argon ions at 300 $\mathrm{K}, 397 \mathrm{~m} / \mathrm{s}$, and $K_{1}=2.41$ (obtained by running the OML/CEC model with $I_{c e c}=0$ ). Equation (19) then give the critical radius in $\mathrm{nm}$ as

$$
r_{N P, \text { crit }}=-0.034 T_{e} \ln \left(5.51 \times 10^{-37} n_{e} T_{e}^{3}\right) .
$$

In summary: in our plasma, with a mix of copper and argon ions, the OML/CEC model applies to copper nanoparticles in the size range $r_{N P} \geq r_{N P, c r i t}$. Here the electron field emission current is much smaller (nominally a factor $K_{2}=10^{-4}$ ) than the OML ion current. For the reference case, the critical radius is $r_{N P, c r i t}=1.43 \mathrm{~nm}$. Although it was derived assuming OML ion current, the condition is still valid (it actually becomes stronger) if there is also a CEC ion current. In that case the total ion current becomes larger, and the EFE current is an even smaller factor.

\subsection{Range discussion}


The condition for the floating potential $\emptyset_{N P}$ is that there is zero net current to the nanoparticle. Since we assume the temperature to be in a range where $I_{T I E}$ is negligible we have:

$$
I_{e}+I_{E F E}+I_{O M L}+I_{C E C}=0 .
$$

For gaining an understanding at which parameter ranges the current contributions are mainly governed by one or another process, it is instructive to write Eq. (21) as a negativecharging current $I_{e}$ that is balanced by the sum of positive-charging currents,

$$
\left|I_{e}\right|=\left|I_{E F E}+I_{O M L}+I_{C E C}\right| .
$$

We define ranges in the nanoparticle radius as where one of the three currents on the right side of Eq. (22) dominates over the other two. This is exemplified in Fig. 6, where Eq. (21) has been solved with the currents taken from Eqs. (1), (4), (7), and (14). For the calculations parameters for the reference case were taken $\left(T_{\mathrm{e}}=1 \mathrm{eV}, n_{\mathrm{e}}=6 \times 10^{18} \mathrm{~m}^{-3}\right.$ and $p=107 \mathrm{~Pa}$ ) and the results are shown in Fig. 6(a) and (c). For comparison, the floating potential and the charging currents were calculated for a higher electron temperature of $T_{\mathrm{e}}=6 \mathrm{eV}$ and are presented in Fig. 6 (b) and (d).

In Fig. 6(a), the floating potential was calculated using Eq. (21) and is shown as a solid black line. The floating potential from OML theory is also shown, as a black dashed line. It is independent of the nanoparticles radius and around -2.8 V. The difference between these two lines is due to CEC which increases the ion current and makes the nanoparticle less negative as compared to OML theory. The influence on the floating potential in the reference case is, however, quite small: only a small deviation of the order of $\Delta \emptyset_{N P}=0.2$ $\mathrm{V}$ is found.

In Fig. 6(c), the contributions of the three positive charging currents $I_{E F E}, I_{O M L}$, and $I_{C E C}$, normalized by the electron collection current $\left|I_{e}\right|$, are separately shown. The currents are indicated as a blue dashed-dotted line for the electron field emission current, a black solid line for the OML current, and a red solid line for the CEC current. Dashed lines separate the relative contributions of copper and argon ions. Looking at the individual contributions, it can be seen that currents of the same order are provided by the argonion current (dashed lines), and the copper-ion current (the difference of the solid and the dashed line). Collisions (the CEC current) contribute to only 10-15\% of the total. The electron field emission is indeed negligible above the critical radius from Eq. (20), 
$r_{N P, \text { crit }}=1.43 \mathrm{~nm}$, but rapidly becomes the largest current with smaller size. The graphs are shaded where $I_{E F E}$ plays a role in order to indicate the uncertainties in using the bulk theory of Murphy and Good [28] to particles of this small size. Furthermore the curves are not drawn below the radius at which the charge becomes - $2 e$ since $I_{E F E}$ is calculated using Eq. (14), which is an approximation valid only for multiple charged nanoparticles.

The effect of a higher electron temperature of $T_{e}=6 \mathrm{eV}$ is shown in Fig. 6(b) and (d). The most significant change is that field emission becomes important at much larger nanoparticle size. The reason for this is that a higher electron temperature gives a higher floating potential, $\emptyset_{N P}=-11 \mathrm{~V}$ as compared to $-2.6 \mathrm{~V}$ in Fig. 6(a). Consequently, the critical radius of Eq. $(20)$ becomes larger, $r_{N P, \text { crit }} \approx 7.5 \mathrm{~nm}$. We can also note that the approach of $\emptyset_{N P}$ towards zero below $4 \mathrm{~nm}$ corresponds to a close to constant value of $E_{N P} \approx\left|\emptyset_{N P}\right| / r_{N P}$. We identify this as the field strength at which $I_{E F E}$ equals the ion collection current.

The two cases shown in Fig. 6 are both in the EFE and in the OML ranges, but they are close to ranges in $r_{N P}$ and $T_{e}$ where the CEC process can become dominant. The CEC ion current of Eq. (8) is proportional to $r_{\text {cap }}^{3}$ and, in the absence of shielding [when Eq. (6) holds], $r_{c a p}$ is proportional to the product $r_{N P}\left|\emptyset_{N P}\right|$. The CEC ion current is therefore in the absence of shielding $\propto\left(r_{N P}\left|\emptyset_{N P}\right|\right)^{3}$ while the OML ion current from Eq. (4) is $\propto$ $r_{N P}^{2}\left|\emptyset_{N P}\right|$. In this case $I_{C E C}$ therefore would increase more rapidly than $I_{O M L}$, with both $r_{N P}$ and potential $\left|\emptyset_{N P}\right|$, and the collection process would go into the CEC range.

The growth rate $d r_{\mathrm{NP}} / d t$ of a nanoparticle is given by Eq. (10). To illustrate some properties of this equation, we neglect the small contribution-in our case about $5 \%$ to the growth by collection of neutrals. Equation (10) then reduces to:

$$
\frac{d r_{\mathrm{NP}}}{d t}=\frac{I_{M^{+} \mathrm{OML}}+{ }_{M^{+}} \mathrm{CEC}}{q_{\mathrm{i}}} \frac{m_{M}}{4 \pi \rho_{M} r_{\mathrm{NP}}^{2}} .
$$

A simplified expression of the growth rate in the OML range, and assuming negligible shielding, was described previously [1]:

$$
\frac{d r_{\mathrm{NP}}}{d t}=n_{\mathrm{i}} v_{\mathrm{th}, \mathrm{i}} \frac{m_{\mathrm{M}}}{\rho_{M}}\left(1+K_{1} \frac{T_{\mathrm{e}}}{T_{\mathrm{i}}}\right)
$$


Instead of solving Eq. (9) for the floating potential, it is here assumed that it can be approximated by Eq. (15). Equation (24) shows that the OML attraction of ions, as compared to the collection of neutral atoms of the same density, increases the growth rate by a factor of $K_{1} T_{e} / T_{i}$. This is a dramatic increase, for our reference case $\approx 100$, and it was demonstrated to open a possibility to steer the growth by controlling $T_{e}$ in the growth zone [1].

\section{Experimental benchmarking}

The growth by collection of ions was studied experimentally by varying the time during which the nanoparticles were kept negative by exposure to a plasma [1]. Details on the experimental setup can be found elsewhere $[1,32]$. In short, growth material is sputtered from a hollow cathode (HC) made of copper, see Fig. 7. The plasma is generated between the hollow cathode and an anode ring located below using high power pulses. The benefit of both using a hollow cathode and high power pulses is a high plasma density and hence a high degree of ionization of the sputtered material. Details on the dynamics of the pulsed plasma including the evolution of the gas temperature were modelled in Ref. [33]. The nanoparticles grow outside of the hollow cathode. The time that the nanoparticles are exposed to a plasma can be varied by changing the distance between the hollow cathode and the anode ring at constant pulse parameters. It was found that when increasing the distance the nanoparticle radius increased from $5 \mathrm{~nm}$ with the anode ring at a position of $30 \mathrm{~mm}$ to $20 \mathrm{~nm}$ with the anode ring located at $60 \mathrm{~mm}$ [1]. The growth rate was estimated from Eq. (24) by calculating the time that a nanoparticle would need to move from $30 \mathrm{~mm}$ to $60 \mathrm{~mm}$ assuming that nanoparticles move together with the gas flow. The time was estimated to $32 \mathrm{~ms}$ resulting in a growth rate of $470 \mathrm{~nm} / \mathrm{s}$ (for details see Ref. [1]). This high growth rate was explained using OML theory, with input parameters for averaged values of the $\mathrm{Cu}$ neutral and ion densities estimated by a separate model developed for the used geometry by Hasan et al [33]. It was proposed that a (time-averaged) electron temperature of $1.7 \mathrm{eV}$ during the growth can explain the fast growth rate, and that 95\% of the growth was due to collection of $\mathrm{Cu}^{+}$ions.

A calculation with the complete OML/CEC model confirms this estimate with only some small corrections. Figure 8 shows the growth from initial seeds of $r_{N P}=5 \mathrm{~nm}$, with similar parameters in the growth zone as used in [1], but with $T_{e}$ varied. The growth of the 
nanoparticles is followed in time by numerical integration of the momentary growth rate $d r_{N P} / d t$ from Eq. (10),

$$
r_{\mathrm{NP}}(t)=r_{0}+\sum_{\tau=0}^{t}\left(\frac{d r_{N P}}{d \tau}\right) \times \Delta \tau
$$

The obtained growth from 5 to $20 \mathrm{~nm}$ radius in $32 \mathrm{~ms}$ is here reproduced at $T_{e}=1.5 \mathrm{eV}$, a slightly lower value than the estimate $1.7 \mathrm{eV}$ from [1]. The same growth in size with a slightly lower $T_{e}$ is possible due to the CEC contribution, $10-15 \%$.

A more pronounced effect of the CEC contribution to growth can be found at lower densities $\left(n_{\mathrm{Cu}^{+}}=3 \cdot 10^{16} \mathrm{~m}^{-3}\right)$ as illustrated in Fig. 9. In this situation, three regions in which the positive charging mechanisms are dominated by contributions of either EFE, OML or CEC [see Fig. 9(a)] appear. The growth of nanoparticles as function of time is shown for different electron temperatures in Fig. 9(b). With increasing electron temperature, a clear effect on the growth rate can be found. However, the total growth rate decreased with decreasing density which results in a much longer growth time for nanoparticles as compared to the case with density of $n_{\mathrm{Cu}^{+}}=3 \cdot 10^{18} \mathrm{~m}^{-3}$.

\section{Summary and Conclusions}

A model is presented for the growth of nanoparticles by collection of ions in a given plasma environment. The model is intended to be a tool for tailoring the plasma parameters in a growth zone so that desired nanoparticles can be produced. The environment is defined by the external parameters: densities and temperatures of the growth material (atoms and ions), the process gas (atoms and ions) and electrons. In the presented general model, charging processes due to collection of ions through OML collection and CEC, electron field and thermionic emission are included. As a limit for the growth of nanoparticles, evaporation is included. The interplay between the processes gives a system of coupled first order differential equations which can be solved iteratively in time from an initial seed nanoparticle size.

In the presented OML/CEC model, the model includes only the parameters and processes needed for OML and CEC. The effects due to evaporation, electron field and thermionic 
emission were only used to, to establish the following limits of applicability for the presented OML/CEC model:

- The pressure must be so low that hydrodynamic effects on the ion collection process can be neglected (Section 2.2.1). We propose the condition $l_{\text {coll }} \gg r_{\text {cap }}$, i.e., that the ion-neutral collision mean free path $l_{\text {coll }}$ is much larger than the capture radius $r_{c a p}$ of Eq. (5). This is satisfied, with a wide margin, in discharges with parameters typical for our nanoparticle synthesis.

- The nanoparticle temperature $T_{N P}$ must be so low that the evaporation rate is much smaller than the growth rate due to the combined OML/CEC ion collection. We have in Section 2.2.2 quantified this condition for the high plasma density and the copper nanoparticles used in the present study. For nanoparticles large enough for bulk evaporation rates to apply, the condition $T_{N P}<1200 \mathrm{~K}$ is assessed as sufficient. For smaller nanoparticles we have made molecular dynamic simulations that show that the evaporation rate is even higher. In this case an even stricter limit on $T_{N P}$ should be obtained.

- The nanoparticle temperature $T_{N P}$ must also be so low that thermionic emission (TIE) of electrons is negligible as compared to all charging particle currents to a nanoparticle from the plasma. This condition is in Section 2.2.3 found to be satisfied already by the temperature limitation $T_{N P}<1200$, mentioned for evaporation. This means, simply, that TIE is negligible in the whole size and temperature range where copper nanoparticles are stable against evaporation: if they do exist, TIE can be neglected.

- The electron field emission current must be negligible as compared to all particle charging currents from the plasma. This gives a condition on the nanoparticle size of the type $r_{N P} \geq r_{N P, c r i t}$. The critical radius $r_{N P, c r i t}$ depends on the plasma density, the electron temperature and the material of the nanoparticle (Section 2.2.4). It is close to proportional to $T_{e}$ and increases, but very slowly, with decreasing plasma density. For copper nanoparticles an approximation of $r_{N P, c r i t}$ is given in analytical 
form in Eq. (20). In our reference case, with $T_{e}=1 \mathrm{eV}$, this gives the condition $r_{N P} \geq$ $r_{N P, \text { crit }}=1.43 \mathrm{~nm}$.

The OML/CEC model was adapted to the parameters of an experiment with observed growth of copper nanoparticles during $32 \mathrm{~ms}$ from 5 to $20 \mathrm{~nm}$ radius [1]. A growth rate in agreement with the experiment was obtained with an average electron temperature of $1.5 \mathrm{eV}$ during the growth. At this temperature the critical radius is $r_{N P, c r i t}=2.1 \mathrm{~nm}$, and consequently the model is within its size range of applicability. The electron temperature of $T_{e}=1.5 \mathrm{eV}$ is slightly lower than an earlier estimate based on a pure OML model [1]. The difference is due to the contribution of CEC ion collection. It is noteworthy that CEC contributes to the growth with about $10 \%$, in spite of the fact that the ion-neutral mean free path is four orders of magnitude larger than the nanoparticle radius, and two orders of magnitude larger than the capture radius of the CEC model.

The growth of a nanoparticle can, even in a time-constant environment, go through a sequence of ranges depending on its momentary size: start with a constant growth rate in the OML regime, then have a phase of accelerating growth rate in the CEC regime, and finally get a strongly reduced growth rate when further expansion of the capture radius is limited by plasma screening. Such a situation can be found for plasmas with ion densities of the order of $10^{16} \mathrm{~m}^{-3}$. The different growth regimes are caused by a variation of the dependency of the growth rate as function of the size of a nanoparticle. From Eqs. (6), (8) and (10) follows that-provided plasma screening of the potential can be neglected-the CEC contribution to this growth rate is proportional to $r_{N P}$. A nanoparticle, for which CEC dominates, will therefore grow at an accelerating rate compared to the pure OML contribution. If it grows to a radius where screening sets in however, $r_{c a p} \approx \lambda_{\mathrm{D}}$, the capture radius begins, according to Eq. (5), to expand more slowly and the growth rate is reduced. A particle for which OML dominates, has instead a growth rate that is independent of the nanoparticle radius up to the size $r_{O M L} \approx \lambda_{D}$. The sizes at which these changes occur depend on the plasma parameters and the gas pressure, which opens possibilities to control the final nanoparticle size.

The first step in further model development is an extension to smaller nanoparticle sizes. This includes a change to a statistical treatment, where the time averaged currents in the 
present work are replaced by probabilities of charge changes of discrete units $e$. Furthermore, the electron field emission will be included into a OML/CEC/EFE model for the charging process. The next step lies further into the future: including $T_{N P}$ as a model parameter, and also all temperature-regulating processes so that the full reaction scheme of Fig. 1 is implemented.

\section{Acknowledgements}

The work was financially supported by the Knut and Alice Wallenberg foundation through grant 2014.0276 and the Swedish Research Council under grant 2008-6572 through the Linköping Linneaus Environment LiLi-NFM. Alexey A. Tal gratefully acknowledges the financial support of the Ministry of Education and Science of the Russian Federation in the framework of Increase Competitiveness Program of MISiS. 


\section{References}

[1] I. Pilch, D. Söderström, M. I. Hasan, U. Helmersson and N. Brenning, "Fast growth of nanoparticles in a hollow cathode plasma through orbit motion limited ion collection," Appl. Phys. Lett., vol. 103, p. 193108, 2013.

[2] J. Allen, "Probe Theory - The Orbital Motion Approach," Physica Scripta, vol. 45, p. 497, 1992.

[3] H. Mott-Smith and I. Langmuir, Phys. Rev., vol. 28, p. 727, 1926.

[4] M. Gatti and U. Kortshagen, "Analytical model of particle charging in plasmas over a wide range of collisionality," Phys. Rev. E, vol. 78, p. 046402, 2008.

[5] I. H. Hutchinson and L. Patacchini, "Computation of the effect of neutral collisions on ion current to a floating sphere in a stationary plasma," Phys. Plasmas, vol. 14, p. 013505, 2007.

[6] S. A. Khrapak and G. E. Morfill, "An interpolation formaula for the ion flux to a small particle in collisional plasmas," Phys. Plasmas, vol. 15, p. 114503, 2008.

[7] S. Khrapak, S. V. Ratynskaia, A. V. Zobnin, A. D. Usachev, V. V. Yaroshenko, M. H. Thoma, M. Kretschmer, H. Höfner, G. E. Morfill, O. Petrov and V. E. Fortov, "Particle charge in the bulk of gas discharges," Phys. Rev. E, vol. 72, p. 016406, 2005.

[8] M. Lampe, R. Goswami, Z. Sternovsky, S. Robertson, V. Gavrishchaka, G. Ganguli and G. Joyce, "Trapped ion effect on shielding, current flow, and charging of a small object in a plasma," Phys. Plasmas, vol. 10, p. 1500, 2003.

[9] A. Piel and C. Schmidt, "Dust charging and charge fluctuations in a weakly collisional radio-frequency sheath at low pressure," Phys. Plasmas, vol. 22, p. 053701, 2015.

[10] T. Zakrzewski and T. Kopiczynski, "Effect of collisions on positive ion collection by a cylindrical Langmuir probe," Plasma Physics, vol. 16, p. 1195, 1974.

[11] F. Galli, Charge and Energy Interactions between Nanoparticles and Low Pressure Plasmas, University of Minnesota: Phd Thesis, 2010.

[12] C. Cui and J. Goree, IEEE Trans. Plasma Sci., vol. 22, p. 151, 1994.

[13] I. B. Denysenko, K. Ostrikov, S. Xu, M. Y. YU and C. H. Diong, J. Appl. Phys., vol. 94, p. 6097, 2003.

[14] S. A. Khrapak, A. V. Ivlev, G. E. Morfill, H. M. Thomas, S. K. Zhdanov, U. Konopka, M.

H. Thoma and R. A. Quinn, Phys. Plasmas, vol. 10, p. 4579, 2003. 
[15] C. Zafiu, A. Melzer and A. Piel, Phys. Plasmas, vol. 10, p. 4582, 2003.

[16] I. H. Hutchinson and C. B. Haakonen, "Collisional effects on nonlinear ion drag force for small grains," Phys. Plasmas, vol. 20, p. 083710, 2013.

[17] J. E. Daugherty, R. K. Porteous, M. D. Kilgore and D. B. Graves, J. Appl. Phys., vol. 72, p. 3934, 1992.

[18] L. M. Chanin and M. A. Biodi, "Mobilities of Mercury ions in Helium, Neon, and Argon," Phys. Rev., vol. 5, p. 1219, 1957.

[19] O. Havnes, G. E. Morfill and C. K. Goertz, "Plasma potential and grain charges in a dust cloud embedded in a plasma," Geophys. Res. Lett. , vol. 89, p. 10999, 1984.

[20] I. Goertz, F. Greiner and A. Piel, "Effects of charge depletion in dusty plasmas," Phys. Plasmas, vol. 18, p. 013703, 2011.

[21] A. Piel, "10.1.6 Influence of Dust Density on Dust Charge," in Plasma Physics: An Introduction to Laboratory, Space, and Fusion Plasmas, Berlin Heidelberg, Springer, 2010, pp. 272-276.

[22] H. R. Maurer and H. Kersten, "On the heating of nano- and microparticles in process plasmas," J. Phys. D: Appl. Phys., vol. 44, p. 174029, 2011.

[23] S. Plimpton, "Fast Parallel Algorithms for Short-Range Molecular Dynamics," J. Comp. Phys., vol. 117, pp. 1-19, 1995.

[24] S. M. Foiles, M. I. Baskes and M. S. Daw, Phys. Rev. B, vol. 33, p. 7983, 1986.

[25] M. Ohring, "The Physics and Chemistry of Evaporation," in Materials Science of Thin Films, Academic Press, 2002, p. 97.

[26] R. E. Honig and D. A. Kramer, "Vapor pressure data for the solid and liquid elements," RCA Review, vol. 30, p. 285, 1969.

[27] S. Dushman, Rev. Mod. Phys., vol. 2, p. 381, 1930.

[28] E. L. Murphy and R. H. Good Jr., Phys. Rev., vol. 102, p. 1464, 1956.

[29] R. C. Weast and M. J. Astle, CRC Handbook of Chemistry and Physics, Boca Raton, Florida, USA: CRC Press Inc., 1980.

[30] J. P. Perdew, J. Phys. B, vol. 37, p. 6175, 1988.

[31] E. Kalered, N. Brenning, I. Pilch, L. Caillault, T. Minea and L. Ojamäe, "On the electron affinity and the charging of clusters in plasmas," no. submitted . 
[32] I. Pilch, D. Söderström, N. Brenning and U. Helmersson, "Size-controlled growth of nanoparticles in a highly ionized pulsed plasma," Appl. Phys. Lett., vol. 102, p. 033108, 2013.

[33] M. I. Hasan, I. Pilch, D. Söderström, D. Lundin, U. Helmersson and N. Brenning, "Modeling the extraction of sputtered metal from high power impulse hollow cathode discharges," Plasma Sources Sci. Technol., vol. 22, p. 035006, 2013.

[34] J. Allen, "Probe Theory - The Orbital Motion Approach," Physica Scripta, vol. 45, p. 497, 1992.

[35] V. V. Kolesnikov, E. V. Polozhentsev, V. P. Sachenko and A. P. Kovtun, "Size fluctuations of the work function in small metallic clusters," Sov. Phys. Solid State, vol. 19, p. 883, 1997.

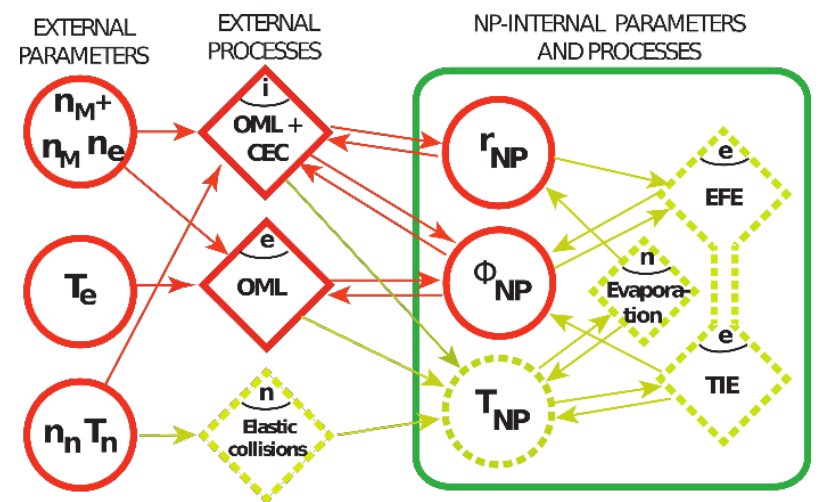

Fig 1. A flow chart for the OML/CEC model. Circles denote parameters and diamonds denote processes. Solid red lines mark parameters and processes that are included in the model. Dashed yellow lines mark parameters and processes that are only treated as needed to assess its limits of applicability. The small $(\sim 5 \%)$ contribution to the growth by collection of neutral metal atoms is not drawn in the figure in order to improve its clarity. 
(a)

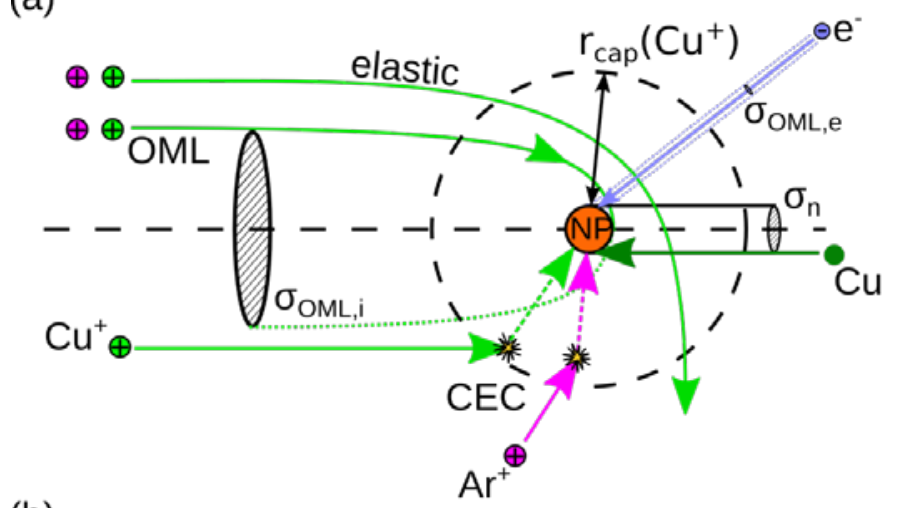

(b)

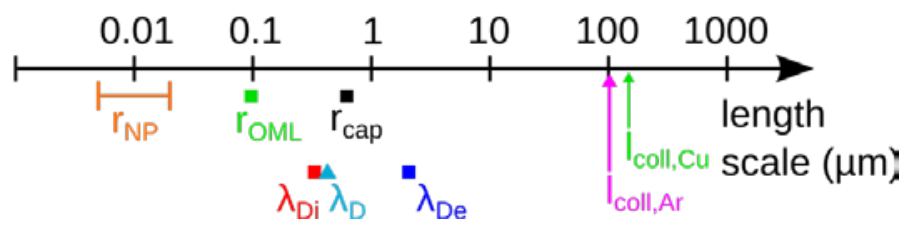

Fig 2. (a) Sketch of the collection processes for orbit motion limited (OML) collection and collision enhanced collection (CEC) of argon $\left(\mathrm{Ar}^{+}\right)$and copper $\left(\mathrm{Cu}^{+}\right)$ions to a negatively charged nanoparticle (NP) with potential $\emptyset_{N P}$. The dashed lines of the CEC processes symbolize what happens between a first ion-neutral collision within the capture radius $r_{\text {cap }}$ and the final capture on a nanoparticle. This process can include a sequence of closed (trapped) orbits and several ionneutral collisions before the ion finally reaches the nanoparticle. (b) Length scales calculated for our reference case with typical parameters from the experiment [1, 33]: $p_{A r}=107 \mathrm{~Pa}, T_{i}=T_{A r}=$ $300 \mathrm{~K}(26 \mathrm{meV}), n_{A r^{+}}=3 \cdot 10^{18} \mathrm{~m}^{-3}, n_{C u^{+}}=3 \cdot 10^{18} \mathrm{~m}^{-3}, n_{e}=6 \cdot 10^{18} \mathrm{~m}^{-3}, T_{e}=1 \mathrm{eV}$ and $r_{N P}=$ $5 \mathrm{~nm}$ to $20 \mathrm{~nm}$. The values of $r_{O M L}$ and $r_{C A P}$ are calculated for $r_{N P}=10 \mathrm{~nm}$. 


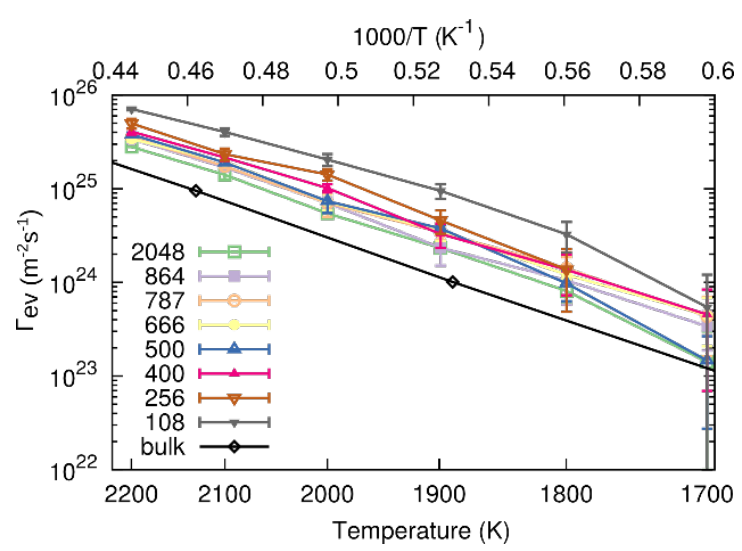

Fig. 3. The evaporation rate as function of temperature, calculated by molecular dynamics simulations, for nanoparticles with cluster size from 108 to 2048 atoms. For comparison, the maximum bulk evaporation rates were calculated using Eq. (11) with data from Ref. [26]. 


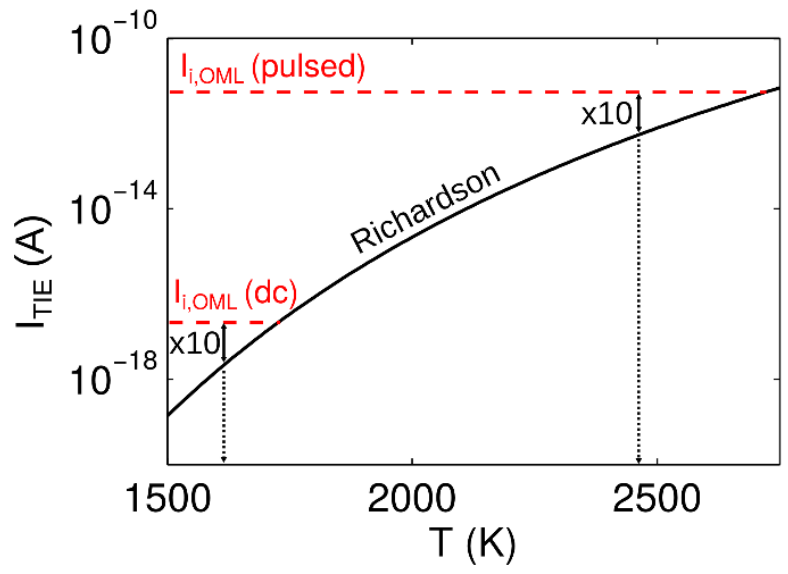

Fig. 4 Thermionic electron emission current according to Richardson-Dushman equation calculated for a nanoparticle radius of $10 \mathrm{~nm}$ (black solid line). The red dashed lines represent lower and upper values estimated for a nanoparticle embedded in a DC plasma $\left(n_{e}=10^{15} \mathrm{~m}^{-3}, T_{e}=1 \mathrm{eV}\right)$ and a pulsed plasma $\left(n_{e}=10^{20} \mathrm{~m}^{-3}, T_{e}=3 \mathrm{eV}\right)$. The black dotted lines indicate temperatures below which the emission current density is a factor 10 smaller than the OML currents to a nanoparticle of the respective case. 


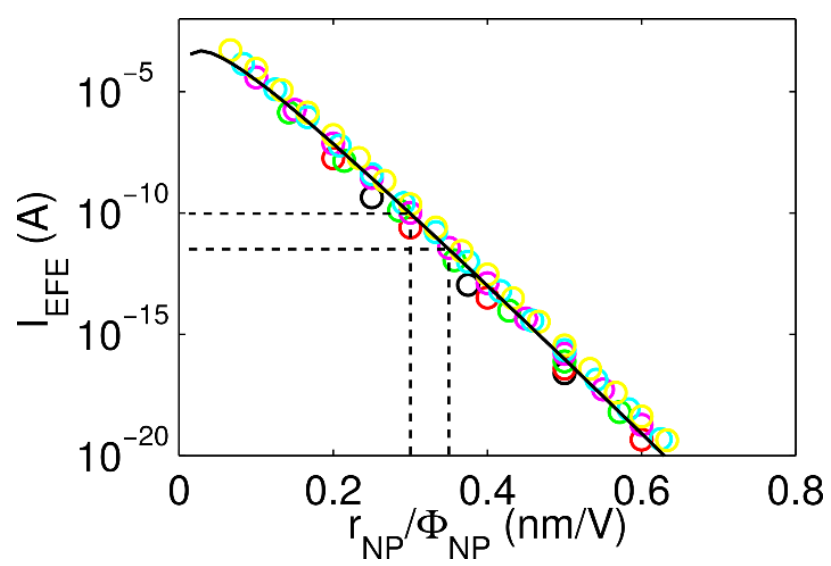

Fig. 5. The electron emission current $I_{E F E}$ from nanoparticles at $300 \mathrm{~K}$ temperature (where thermionic emission is negligible) as function of the normalized radius $r_{N P} /\left|\emptyset_{N P}\right|$. The data points are simulated values for given nanoparticle potentials: $-4 \mathrm{~V}$ (black circles), $-5 \mathrm{~V}$ (red circles), $-7 \mathrm{~V}$ (green circles), -10 $\mathrm{V}$ (magenta circles), $-12 \mathrm{~V}$ (cyan circles), and $-15 \mathrm{~V}$ (yellow circles). The solid line is a fitted curve [Eq. (14)] to estimate the field emission current. The dashed black lines show that a variation in electron emission currents by several orders of magnitude correspond to only small variations in the normalized radius $r_{N P} /\left|\emptyset_{N P}\right|$. 

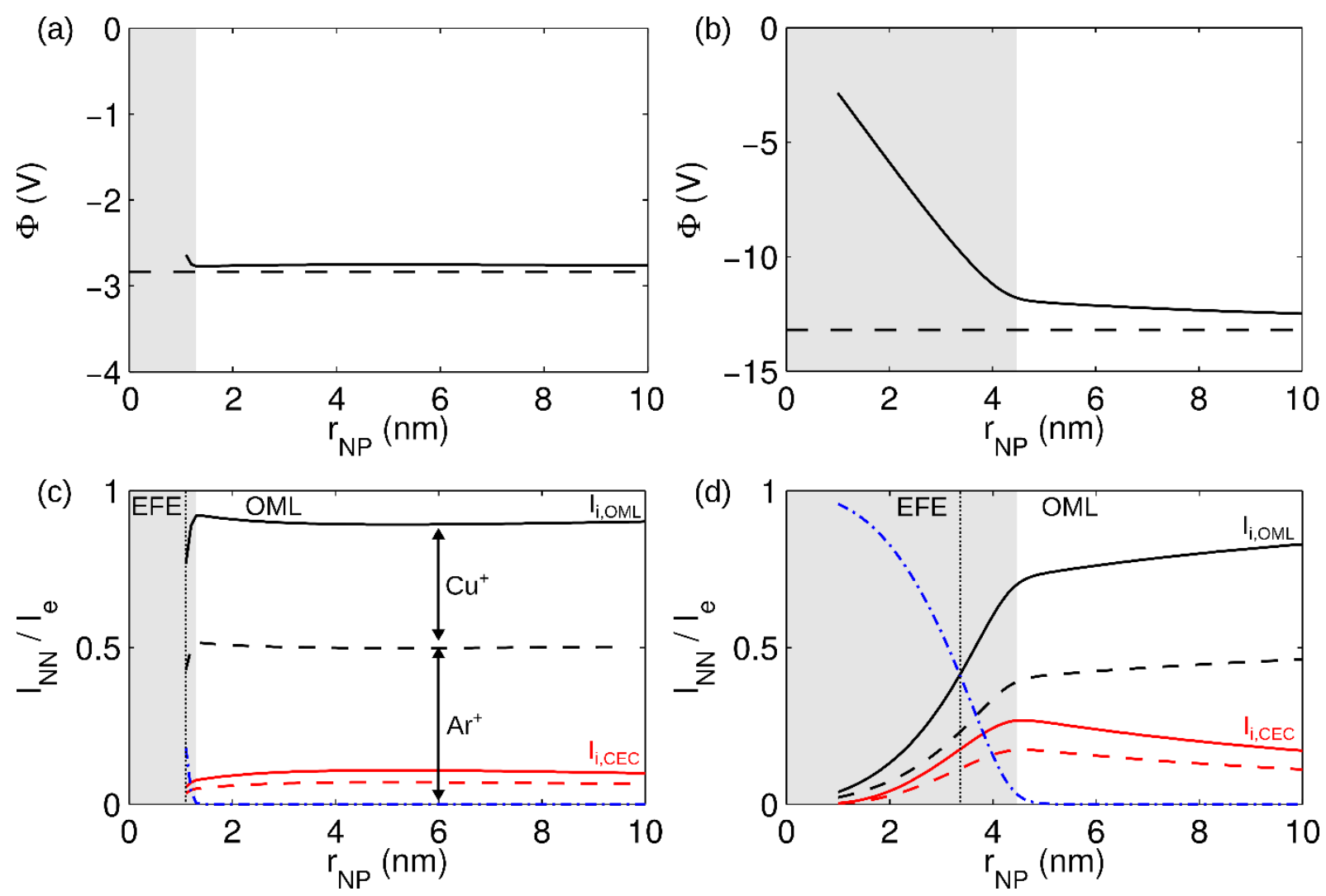

Fig. 6. (a, b) Potential of a nanoparticle calculated as function of radius assuming OML theory (black dashed line) including the collisional currents and electron field emission (black solid line). The curves were calculated for (a) the reference case $\left(p_{A r}=107 \mathrm{~Pa}, T_{i}=T_{A r}=26 \mathrm{meV}, n_{A r^{+}}=3 \cdot 10^{18}\right.$ $\mathrm{m}^{-3}, n_{\mathrm{Cu}^{+}}=3 \cdot 10^{18} \mathrm{~m}^{-3}, n_{e}=6 \cdot 10^{18} \mathrm{~m}^{-3}, T_{e}=1 \mathrm{eV}$ ) and (b) a higher electron temperature of $T_{e}=$ $6 \mathrm{eV}$. The plot is shaded in the range where the calculated potential is uncertain, both because the analytical expression of Murphy and Good [5] becomes uncertain and because a statistical model should be used with discrete charges in units $e$. (c, d) Individual currents calculated with the OML/CEC model for (a) the reference case and (b) a higher electron temperature of $T_{e}=6 \mathrm{eV}$. The positive charging currents for electron field emission $I_{E F E}$ (blue dashed-dotted line), orbital motion limited ion current $I_{i, O M L}$ (OML, black lines) and collision-enhanced collection ion current $I_{i, C E C}$ (CEC, red lines) are all normalized to the electron current $I_{e}$. The dashed lines represent contribution of argon ions to the total current contribution (solid lines), and the fraction of copper ions contributing to the total is given by the difference of the solid and the dashed curves. The plot is shaded in the range where the result is uncertain as discussed in the text. 

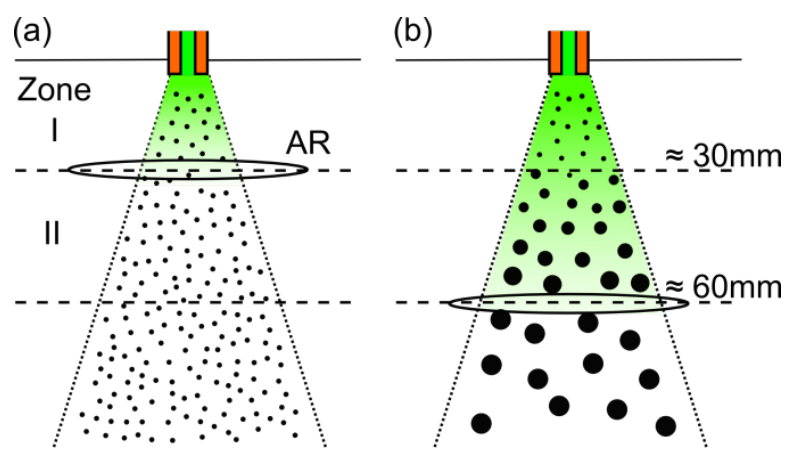

Fig. 7. Sketch of the experimental geometry for investigating the hypothesis of fast growth by ion collection. Growth material is sputtered from a hollow cathode made of copper in the top and nanoparticles form in the region outside the hollow cathode. By varying the position of an anode ring (AR), the time of plasma exposure while growth can be controlled, which is indicated for (a) an upper position around $30 \mathrm{~mm}$ and (b) a lower position around $60 \mathrm{~mm}$. 


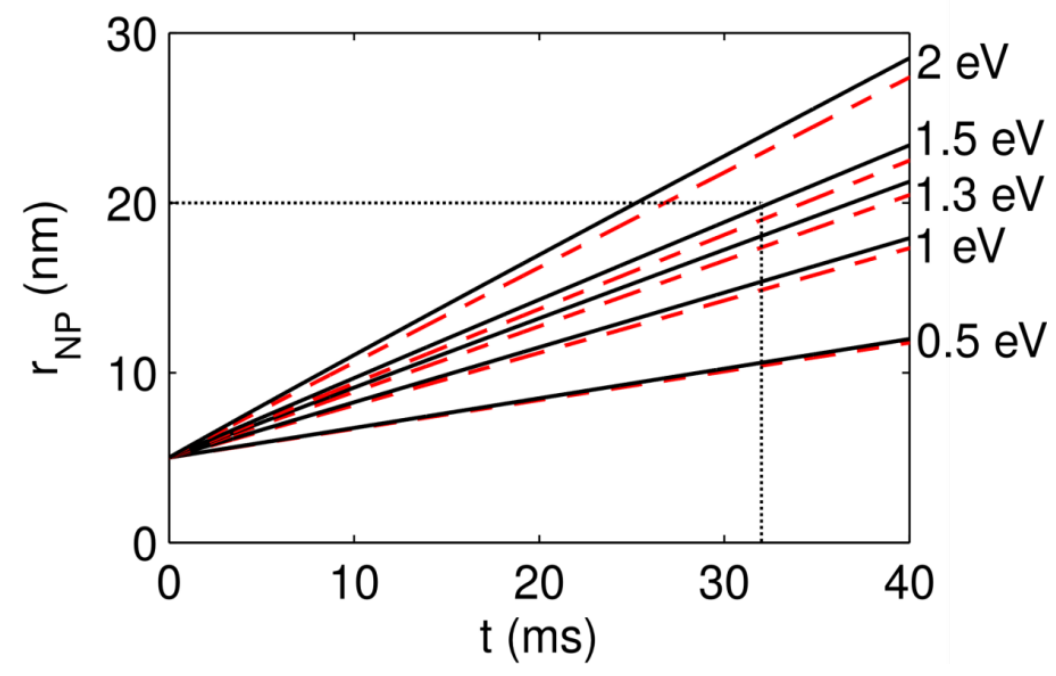

Fig 8. The growth of nanoparticles is modelled as function of time and for different electron temperatures. Solid black lines were calculated using Eq. (21) for the OML/CEC model and dashed red lines were calculated using Eq. (24) taking only the contribution from the OML model into consideration. At the time $t=t_{0}=0$, the nanoparticles start with a radius of $5 \mathrm{~nm}$ at the position $z=30 \mathrm{~mm}$ (see Fig. 7). According to experimental estimations [1], a nanoparticle has grown to a size of $20 \mathrm{~nm}$ after passing the anode ring position at $z=60 \mathrm{~mm}$, which corresponds to a travel time of $t=t_{0}+32$ ( $\mathrm{ms}$ ) between the anode ring position at 30 and $60 \mathrm{~mm}$. The size increase is reproduced by the OML/CEC model for an electron temperature of $T_{e}=1.5 \mathrm{eV}$. 

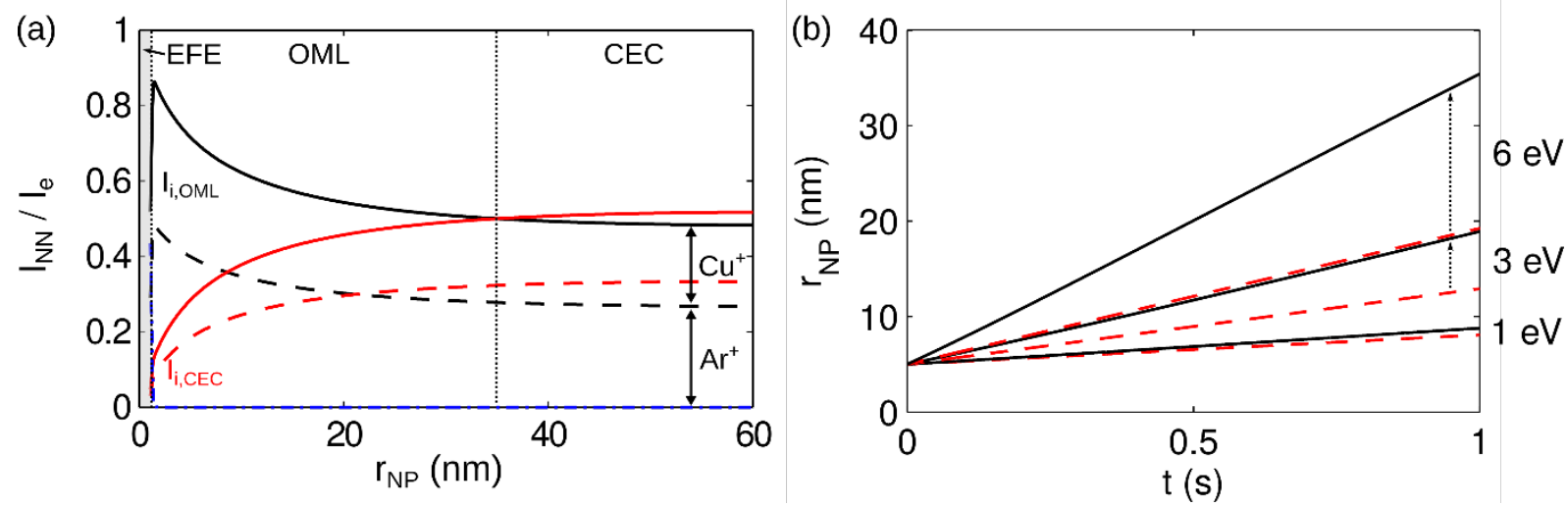

Fig 9. (a) Positive charging currents to a nanoparticle calculated at an electron temperature of $T_{e}=1 \mathrm{eV}$ and for lower densities $\left(n_{\mathrm{Cu}^{+}}=3 \cdot 10^{16} \mathrm{~m}^{-3}\right.$ and $\left.n_{e}=6 \cdot 10^{16} \mathrm{~m}^{-3}\right)$ compared to the standard parameter. Under those conditions, three regions are found in which the charging currents are dominated by EFE, OML or CEC. (b) The growth of nanoparticles was calculated as function of time for different electron temperatures. The solid black lines represent the OML/CEC model and the dashed red lines the OML model. At high electron temperatures a clear increase of the growth rate is found which is ascribed to the additional contribution of $\mathrm{CEC}$, as indicated by the dashed black arrows. 


\section{Supplementary information}

The growth rate for the OML model can be approximated when the electron-to-ion temperature ratio was defined using the following equation [1]:

$$
\frac{d r_{N P}}{d t}=\frac{1}{4} n_{i} v_{t h} \frac{m_{i}}{\rho}\left(1+K \frac{T_{e}}{T_{i}}\right)
$$

For argon ions and a temperature ratio of $T_{e} / T_{i}=100$, the constant $K$ is 2.41 .

In Fig.1 of the supplementary information, the growth rate is shown as function of electron temperature for the OML approach using the approximated equation (black dashed line) and without the approximation (solid black line), where the floating potential was calculated by solving the floating condition: $I_{e, O M L}+I_{i, O M L}=0$. The approximated growth rate underestimates the growth rates for temperature ratios $T_{e} / T_{i}$ below $100\left(T_{i}=0.026 \mathrm{eV}\right)$ and overestimates the growth rates above 100 . In the temperature range between 1 and $5 \mathrm{eV}$, the approximation gives a fair agreement.

For the collisional approach, the growth rate was calculated using Eq. (23) of the manuscript for three constant nanoparticle sizes: $5 \mathrm{~nm}$ (green dashed-dotted line), $10 \mathrm{~nm}$ (red long-dashed line) and $20 \mathrm{~nm}$ (blue, dashed line). The OML currents without approximation and the collisional approach give similar growth rates at low electron temperatures below $0.1 \mathrm{eV}$. Even at higher electron temperatures, the deviation is small in the logarithmic representation.

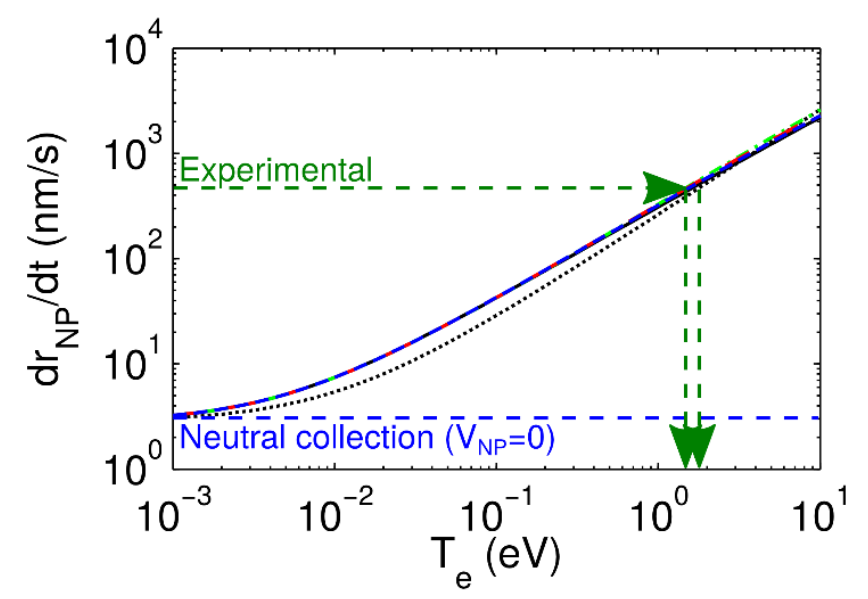

Fig. 1: Growth rate as function of electron temperature.

\section{Reference}

[1] I. Pilch, D. Söderström, M. I. Hasan, U. Helmersson and N. Brenning, Appl. Phys. Lett. 103, 193108 (2013). 